\title{
Factors associated with unfavorable evolution of tuberculosis in HIV infected persons
}

\author{
Florentina Dumitrescu ${ }^{1,2^{*}}$, Andreea Cristina Stoian ${ }^{1,2}$, Augustin Cupşa ${ }^{1,2}$, Mihai Jianu ${ }^{2}$, Alina Lungu ${ }^{2}$, \\ Alexandra Corobea ${ }^{2}$, Dina Maria Cupşa ${ }^{2}$, Adrian Neguleț ${ }^{2}$ \\ From The 9th Edition of the Scientific Days of the National Institute for Infectious Diseases Prof Dr Matei Bals \\ Bucharest, Romania. 23-25 October 2013
}

\section{Background}

Tuberculosis (TB) is one of the most common opportunistic infections in HIV-infected patients, with severe evolutive potential.

We evaluated the prevalence and the clinical aspects of TB in HIV-infected persons in order to identify factors associated with unfavorable evolution of TB.

\section{Methods}

We performed an observational, retrospective study (01 January 2009 - 31 December 2011) on 387 HIV-infected patients in evidence at the HIV/AIDS Craiova Regional Center. We analyzed the epidemiological, clinical and paraclinical data (smears and cultures for Mycobacterium tuberculosis, immunovirological evaluation) for patients who presented at least 2 times per year at the regional center.

\section{Results}

During the studied period 59 patients (15.3\%) presented TB (9 cases simultaneously diagnosed with HIV and TB). General data on the study group: average age at the moment of TB diagnosis: $25.5 \pm 7.4$ years; equal gender distribution: male/female 30/29; rural/urban 40/19 (67.8/32.2\%); average CD4 count 3 months before TB diagnosis: $179 \pm 204$ cells/ $\mathrm{cmm}$; average viral load: $5.09 \pm 5.3 \log _{10}$. TB was: pulmonary: 46 cases $(77.9 \%)$, extrapulmonary 8 (13.6\%), multiple locations 5 (8.5\%). 19 cases (32.2\%) were bacteriologically confirmed (positive smears and/or positive cultures). 53 patients (89.8\%) were under antiretroviral treatment, with a very good adherence in 19 patients (32.2\%). Other opportunistic infections apart from TB were recorded in 28 patients (47.4\%). 29 patients (49.2\%) had favorable outcome, complication or relapse occurred in 10 cases (16.9\%) and 20 patients (33.9\%) died. Factors associated with unfavorable evolution were: delay in the introduction of anti-TB treatment more than 30 days from the first symptom $(p=0.001)$; the presence of other opportunistic infections apart from TB ( $\mathrm{p}=0.0001)$, low adherence to treatment $(\mathrm{p}=0.0001)$. Death was associated with extrapulmonary/ multiple location of TB $(\mathrm{p}=0.005)$ and average $\mathrm{CD} 4<100$ cells $/ \mathrm{cmm}(\mathrm{p}=0.002)$.

\section{Conclusion}

Tuberculosis is common in patients infected with HIV, the unfavorable evolution being associated with severe immunosuppression, extrapulmonary ТВ location, poor adherence and delay in the introduction of anti-TB treatment.

\section{Authors' details}

"University of Medicine and Pharmacy Craiova, Romania. "2Victor Babeş" Clinical Hospital of Infectious Diseases and Pneumology, Craiova, Romania.

Published: 16 December 2013

doi:10.1186/1471-2334-13-S1-P16

Cite this article as: Dumitrescu et al:: Factors associated with unfavorable evolution of tuberculosis in HIV infected persons. BMC Infectious Diseases 2013 13(Suppl 1):P16. 